\title{
You can recognize the shape of a figure from its shadows!
}

\author{
ÁrPÁd KURUSA*
}

\begin{abstract}
Let $\mathcal{C}_{1}$ and $\mathcal{C}_{2}$ be convex closed domains in the plane with $\mathrm{C}^{2}$ boundaries $\partial \mathcal{C}_{1}$ and $\partial \mathcal{C}_{2}$ intersecting each other in nonzero angles. Assume the two strictly convex bodies $\mathcal{F}_{1}$ and $\mathcal{F}_{2}$ with $\mathrm{C}^{2}$ boundaries in the interior of $\mathcal{C}_{1} \cap \mathcal{C}_{2}$ subtend equal visual angles at each point of $\partial \mathcal{C}_{1}$ and $\partial \mathcal{C}_{2}$. Then $\mathcal{F}_{1}$ and $\mathcal{F}_{2}$ coincide. Generalizations are also discussed.
\end{abstract}

\section{Introduction}

The already classical question of P.C. Hammer 'How many X-ray pictures of a convex body must be taken to permit its exact reconstruction?' has been investigated in several aspects during the last decade $[2,3,10,12]$. Motivated by the results the problem arises if the reconstruction is possible using poorer pictures. J. Kincses [6] proposed to investigate the shadow picture.

The shadow picture of a convex body is closely related to the X-ray picture, but, somewhat surprisingly, it has been defined only for the parallel beam case. The parallel beam shadow picture corresponding to a direction is the orthogonal projection of the body to the subspace orthogonal to the given direction. One can regard this as the parallel beam X-ray picture of an impenetrable body. In the plane it is the width of the body in the given direction. Therefore the reconstruction is impossible as there are different convex bodies (bodies of constant width for example), even polygons, having equal widths in every directions.

We define the divergent beam shadow picture of a convex body in the plane as the divergent beam X-ray picture of an impenetrable convex body $\mathcal{F}$. More exactly, the shadow picture of $\mathcal{F}$ at $P \in \mathbb{R}^{2}$ is the visual angle of $\mathcal{F}$ at $P$, i.e. the angle the body $\mathcal{F}$ subtends at $P$. We disregard the position of the angle and consider only

AMS Subject Classification (2000): 0052,0054.

* Supported by the Hungarian NSF, OTKA Nr. T4427

Geom. Dedicata, 59 (1996), 113-125.

(c) Á. Kurusa 
its measure. The somewhat more complicated definition for higher dimensions is placed to Section 3 .

Question. 'On what point-set the divergent beam shadow picture of a convex body has to be known to permit its exact reconstruction?'.

Obviously, no finite set is enough. According to Green's result [4], the set of points of some curve may be enough to determine some bodies. He proved that a convex body subtending a constant angle on a circle must be a circle except certain types of the angle. J. Kincses and the author proved in [6] that the convex polygons can be distinguished from each other by their visual angles taken on virtually any curve.

J.C.C. Nietsche [8] proved that a set subtending constant angles on two concentric circles must be a circle. This and Falconer's result [2] motivated the following result.

Theorem 1. Let $\mathcal{C}_{1}, \mathcal{C}_{2}, \mathcal{F}_{1}$ and $\mathcal{F}_{2}$ be closed convex domains with $\mathrm{C}^{2}$ boundaries in the plane. The strictly convex bodies $\mathcal{F}_{1}$ and $\mathcal{F}_{2}$ are in the interior of $\mathcal{C}_{1} \cap \mathcal{C}_{2}$. The boundaries of $\mathcal{C}_{1}$ and $\mathcal{C}_{2}$ intersect each other in nonzero angles. If $\mathcal{F}_{1}$ and $\mathcal{F}_{2}$ subtend equal visual angles at each point of $\partial \mathcal{C}_{1} \cup \partial \mathcal{C}_{2}$ then they coincide.

Our proof, given in the next section, resembles Falconer's method in [2] and also uses some ideas of Rogers [10]. It is mainly based on geometric observations and on some analysis. A closer look at the proof indicates a kind of duality to the $\mathrm{X}$-ray problem for two point sources [2]. T. Ódor [9] kindly informed the author that this duality leads to a variant of the X-ray problem with two point sources. The intersection condition in Theorem 1 seems to be unavoidable disregarding the cases in [7].

In Section 3 we discuss the related problem in higher dimensions and, after defining the shadow picture for higher dimensions, we prove an easy but interesting uniqueness result. Then we generalize the notion of the visual angle to general curves in the plane and formulate a conjecture for this case.

The author wishes to thank the Soros Foundation (New York) for supporting his visit at the MIT's Math. Department, where this paper was born. Thanks are also due to the Department of Mathematics of MIT and to the Matematische Institut of the Universität Erlangen-Nürnberg for the support and assistance in finishing this paper. I thank the referee for a number of improvements on the form of this paper.

Geom. Dedicata, 59 (1996), 113-125.

(c) Á. Kurusa 


\section{The proof}

Theorem 1 will be proved through an easy lemma and a rather complicated proposition. Our notations throughout these are the following. $\mathcal{F}$ is a strictly convex body in the plane with $\mathrm{C}^{2}$ boundary. The $\mathrm{C}^{2}$ curve $g:[-1,0] \rightarrow \mathbb{R}^{2}$ is outside $\mathcal{F}$, parameterized by arc length and its tangents do not meet $\mathcal{F} . T(s), t(s)$, $n(s)$ and $\kappa(s)$ are the tangent line, the tangent vector, the normal vector and the curvature of the curve $g$ at $g(s)$, respectively. $\nu(s)$ denotes the visual angle of $\mathcal{F}$ at the point $g(s) . \mathcal{T}^{a}(s)$ and $\mathcal{T}^{b}(s)$ denote the tangent lines of $\mathcal{F}$ through $g(s) . \tau^{a}(s)$ and $\tau^{b}(s)$ denote the unit direction vectors of these tangents lines, respectively. They are in the same side of $T(s)$ as $\mathcal{F}$ and $\tau^{a}(s)$ is a positive linear combination of $\tau^{b}(s)$ and $t(s) . \alpha(s)$ and $\beta(s)$ are the angles of $\tau^{a}(s)$ and $\tau^{b}(s)$ to $t(s)$, respectively, so that $0<\alpha(s)<\beta(s)<\pi$. $\kappa^{a}(s)$ and $\kappa^{b}(s)$ are the curvatures of $\partial \mathcal{F}$ at the points $A(s)$ and $B(s)$, the intersection of $\mathcal{F}$ with the tangent lines $\mathcal{T}^{a}(s)$ and $\mathcal{T}^{b}(s)$, respectively. Let $a(s)=|A(s)-g(s)|$ and $b(s)=|B(s)-g(s)|$. If more objects enter the picture, then we shall index them consequently. If no argument of a function is written then it is understood at the appropriate arclength parameter $s \in[-1,0]$.

The second equation in the following lemma will be used only to see that $\ddot{\nu}$ is sufficiently smooth. Therefore we only outline its proof.

Lemma 1. With the notations introduced above,

$\dot{\nu}=\frac{\sin \beta}{b}-\frac{\sin \alpha}{a} \quad$ and $\quad \ddot{\nu}=\frac{\sin 2 \beta}{b^{2}}-\frac{\sin 2 \alpha}{a^{2}}+\frac{\sin ^{2} \beta}{b^{3} \kappa^{b}}+\frac{\sin ^{2} \alpha}{a^{3} \kappa^{a}}-\kappa\left(\frac{\cos \beta}{b}-\frac{\cos \alpha}{a}\right)$

Proof. Only the derivatives of $\beta$ will be calculated. The corresponding results for $\alpha$ can be obtained in the same way. We use row-vectors and the rotation matrix

$$
\left(\begin{array}{cc}
\cos \xi & \sin \xi \\
-\sin \xi & \cos \xi
\end{array}\right)=R(\xi)
$$

Obviously $B(s)=b(s) t(s) R(\beta(s))+g(s)$. Differentiating w.r.t. $s$, we obtain

$$
\dot{B}=\dot{b} t R(\beta)+b \kappa n R(\beta)+b t \dot{R}(\beta) \dot{\beta}+t
$$

The vector $\dot{B}$ is tangent to $\mathcal{F}$, hence $\dot{B}=-|\dot{B}| t R(\beta)$. Since $t \dot{R}(\beta)=n R(\beta)$, the multiplication of the above equation with $R(-\beta)$ gives

$$
t R(-\beta)=-(|\dot{B}|+\dot{b}) t-b(\kappa+\dot{\beta}) n
$$

Geom. Dedicata, 59 (1996), 113-125.

(c) Á. Kurusa 
Also $t R(-\beta)=t \cos \beta-n \sin \beta$ is valid, therefore

$$
|\dot{B}|+\dot{b}=-\cos \beta \quad \text { and } \quad b(\kappa+\dot{\beta})=\sin \beta .
$$

The second equation proves our first aim by repeating the same argument for $\alpha$.

For our second claim we calculate the angle between $\mathcal{T}^{b}(s)$ and $\mathcal{T}^{b}(s+h)$ on $\partial \mathcal{F}$ and also on $g$ that results in

$$
\int_{i(s)}^{i(s+h)} \kappa^{b} \mathrm{~d} i=\beta(s+h)-\beta(s)+\int_{0}^{h} \kappa(s+v) \mathrm{d} v
$$

where $i$ is the arclength parameter on $\partial \mathcal{F}$. Differentiating this with respect to $h$ and then taking $h=0$ we obtain $\kappa^{b}|\dot{B}|=\kappa+\dot{\beta}$, because $|\dot{B}|=\frac{\mathrm{d} i}{\mathrm{~d} s}$. Combining this with $(*)$ we get $\dot{b}=-\cos \beta-\frac{\sin \beta}{b \kappa^{b}}$. Substituting this and the similar equation for $\alpha$ into the derivative of the first equation of the lemma one leads to the second one.

Note also, that the first claim of the lemma is valid also for $\mathrm{C}^{1}$ curves: calculating $\dot{\beta}$ and $\dot{\alpha}$ simultaneously, the terms otherwise leading to $\kappa$ cancel out.

The key to Theorem 1 is in the following proposition. We have two $\mathrm{C}^{2}$ curves $g_{1}$ and $g_{2}$ and all the other objects are indexed accordingly.

Proposition 1. Let $g_{1}(0)=g_{2}(0)=X,\left|t_{1}(0) \times t_{2}(0)\right|=\sin \gamma \neq 0$. Then the visual angle functions $\nu_{1}$ and $\nu_{2}$ determine the function $\beta_{1}$ on $(-\varepsilon, 0]$ for $\varepsilon>0$ small enough.

Because of the complicated calculations needed, it is worthwile surveying our method. First observe that $\tau_{i}^{a}$ and $\tau_{i}^{b}$ determines each other by rotating the given one around $g_{i}(s)$ with the angle $\nu_{i}(s)$ in the appropriate direction. It is also clear, that a tangent of $\mathcal{F}$ close enough to $X$ intersects both curves.

Thus, starting from one tangent, we can determine two infinite sequences of tangents of $\mathcal{F}$ : simply rotate the tangent(s) around its (their) intersections with the curves by the known visual angle, and then repeat. Since the rotation always leads to new tangents of $\mathcal{F}$, the iteration gives the infinite sequences of tangents. The appropriate sequences will converge to the tangents through $X$.

A similar infinite sequence can be obtained from an arbitrary straight line, sufficiently close to a tangent. Simply rotate the straight line around its intersections with the curves by the visual angles given at the intersections, and then iterate this step. First, we shall prove, that this sequence converges to a straight line through $X$. Then we show, that this limit line is the tangent of $\mathcal{F}$ if and only if the original straight line was a tangent of $\mathcal{F}$. This gives the statement of the proposition.

Geom. Dedicata, 59 (1996), 113-125.

(c) Á. Kurusa 
Proof. To simplify notation we set $\alpha=\alpha_{1}(0)=\alpha_{2}(0)+\gamma, \beta=\beta_{1}(0)=\beta_{2}(0)+\gamma$, $\nu=\nu_{1}(0)=\nu_{2}(0), a=a_{1}(0)=a_{2}(0)$ and $b=b_{1}(0)=b_{2}(0)$, and assume $0<\gamma \leq$ $\pi / 2$. Then $g_{1}$ separates $B_{1}$ and the intersection $\mathcal{T}_{1}^{b} \cap g_{2}$, if this exists.

Since

$$
\operatorname{det}\left(\begin{array}{cc}
\sin \alpha & \sin \beta \\
\sin (\alpha+\gamma) & \sin (\beta+\gamma)
\end{array}\right)=\sin \nu \sin \gamma \neq 0
$$

the determinant of the system of equations $x \sin \alpha_{i}(0)-y \sin \beta_{i}(0)=0(i=1,2)$ is not zero, hence we may suppose $a \sin \alpha_{1}(0)-b \sin \beta_{1}(0) \neq 0$.

By the assumptions there exists $\varepsilon_{0}>0$ such that for $-\varepsilon_{0}<s \leq 0$ the tangent $\mathcal{T}_{1}^{b}(s)$ cuts $g_{2}$ in the interval $[-1 / 2,0]$. Let $\delta_{0}>0$ be such that any of the straight lines through any point $g_{1}(s)$ with $-\varepsilon_{0}<s \leq 0$ making angle with $\mathcal{T}_{1}^{b}(s)$ smaller than $\delta_{0}$ cuts $g_{2}$ in the interval [-3/4,0]. Let $\varepsilon_{0}>\varepsilon_{1}>0$ be such that for $-\varepsilon_{1}<s \leq 0$ the angles $\tau_{1}^{b}(s) \tau_{1}^{b}(0) \angle$ and $\tau_{1}^{a}(s) \tau_{1}^{a}(0) \angle$ are smaller than $\delta_{1}=\delta_{0} / 4$. We restrict our calculation to the interval $\left(-\varepsilon_{1}, 0\right)$ and to the set $S(s)$ of the straight lines through $g_{1}(s)$ making angle with $\mathcal{T}_{1}^{b}(s)$ smaller than $\delta_{1}$.

We define a map $f$ from $S\left(s_{0}\right)$ into the set of straight lines through $X$ in the following way. Let $l_{0} \in S\left(s_{0}\right)$. By the above conditions, it cuts $g_{2}$ in a point, say, $g_{2}\left(r_{0}\right)$. Rotate $l_{0}$ around $g_{2}\left(r_{0}\right)$ clockwise with angle $\nu_{2}\left(r_{0}\right)$ to get $l_{1} \cdot l_{1}$ must intersect $g_{1}$ in a point, say $g_{1}\left(s_{1}\right)$, so that $s_{0}<s_{1}$. Rotate $l_{1}$ around $g_{1}\left(s_{1}\right)$ anticlockwise with angle $\nu_{1}\left(s_{1}\right)$ to get $l_{2}$. Repeat this procedure with $l_{2}$ to get first $l_{3}$ and $r_{1}$ and then $l_{4}$ and $s_{2}$ and so on. In this way, we get an infinite sequence $\left\{l_{i}\right\}_{i=0}^{\infty}$ of straight lines.

We prove that the limit line $l=\lim _{i \rightarrow \infty} l_{2 i}$ exists and goes through $X$. Then we define $f\left(l_{0}\right):=l$.

Let $\omega_{i}$ be the angle of $l_{2 i}$ to $t_{1}\left(s_{i}\right)$. Then

$$
\omega_{i+1}=\omega_{i}-\nu_{2}\left(r_{i}\right)+\nu_{1}\left(s_{i+1}\right)-\int_{s_{i}}^{s_{i+1}} \kappa_{1}(s) \mathrm{d} s
$$

because the tangent $t_{1}$ of $g_{1}$ rotates along $g_{1}$ with speed $\kappa_{1}$ and the straight line $l_{2 i}$ has been rotated with $-\nu_{2}\left(r_{i}\right)$ and then with $\nu_{1}\left(s_{i+1}\right)$. By the previous lemma, we can expand $\nu_{1}$ and $\nu_{2}$ into a Taylor series of second order around the zero that gives

$$
\omega_{i+1}=\omega_{i}-r_{i} \dot{\nu}_{2}(0)-r_{i}^{2} O(1)+s_{i+1} \dot{\nu}_{1}(0)+s_{i+1}^{2} O(1)-\int_{s_{i}}^{s_{i+1}} \kappa_{1}(s) \mathrm{d} s
$$

where $O$ is the usual Landau symbol, meaning that $\lim _{i \rightarrow \infty} O(1)=c$ for some constant $c$.

Geom. Dedicata, 59 (1996), 113-125.

(c) Á. Kurusa 
By the following inequalities the sequence $l_{2 i}$ keeps close to $\tau_{1}^{b}(0)$. We shall use this fact frequently.

$$
\begin{aligned}
\left|\omega_{i}-\beta\right| & \leq\left|\omega_{0}-\beta_{1}\left(s_{0}\right)\right|+\left|\beta_{1}\left(s_{i}\right)-\beta\right|+\sum_{j=1}^{i}\left|\left(\omega_{j}-\beta_{1}\left(s_{j}\right)\right)-\left(\omega_{j-1}-\beta_{1}\left(s_{j-1}\right)\right)\right| \\
& \leq 2 \delta_{1}+\sum_{j=1}^{i}\left|\tau_{1}^{b}\left(s_{j-1}\right) \tau_{2}^{b}\left(r_{j-1}\right) \angle-\tau_{1}^{a}\left(s_{j}\right) \tau_{2}^{a}\left(r_{j-1}\right) \angle\right| \\
& \leq 2 \delta_{1}+\sum_{j=1}^{i} \tau_{1}^{b}\left(s_{j-1}\right) \tau_{2}^{b}\left(r_{j-1}\right) \angle+\sum_{j=1}^{i} \tau_{1}^{a}\left(s_{j}\right) \tau_{2}^{a}\left(r_{j-1}\right) \angle \\
& \leq 2 \delta_{1}+\tau_{1}^{b}\left(s_{0}\right) \tau_{2}^{b}\left(r_{i}\right) \angle+\tau_{1}^{a}\left(s_{1}\right) \tau_{2}^{a}\left(r_{i-1}\right) \angle \leq 2 \delta_{1}+\delta_{1}+\delta_{1}=\delta_{0} .
\end{aligned}
$$

For the second inequality we used that

$$
\begin{aligned}
\omega_{j}-\beta_{1}\left(s_{j}\right) & =l_{2 j} \tau_{1}^{b}\left(s_{j}\right) \angle=l_{2 j-1} \tau_{1}^{a}\left(s_{j}\right) \angle=\tau_{1}^{a}\left(s_{j}\right) \tau_{2}^{a}\left(r_{j-1}\right) \angle+l_{2 j-1} \tau_{2}^{a}\left(r_{j-1}\right) \angle \\
\omega_{j-1}-\beta_{1}\left(s_{j-1}\right) & =\tau_{1}^{b}\left(s_{j-1}\right) \tau_{2}^{b}\left(r_{j-1}\right) \angle+l_{2 j-2} \tau_{2}^{b}\left(r_{j-1}\right) \angle
\end{aligned}
$$

and that $l_{2 j-1} \tau_{2}^{a}\left(r_{j-1}\right) \angle=l_{2 j-2} \tau_{2}^{b}\left(r_{j-1}\right) \angle$.

Our further observation is that the sequences $s_{i}$ and $r_{i}$ are increasing and bounded, hence $s=\lim s_{i}$ and $r=\lim r_{i}$ exist. Assume $g_{1}(s) \neq g_{2}(r)$. This implies the existence of the limit straight lines $l^{b}=\lim l_{2 i}$ and $l^{a}=\lim l_{2 i+1}$. Then $l^{b} \equiv l^{a}$, because both go through $g_{1}(s)$ and $g_{2}(r)$. This contradicts that the angle between $l_{2 i}$ and $l_{2 i+1}$ is $\nu_{2}\left(r_{i}\right) \rightarrow \nu_{2}(r)>0$, hence $g_{1}(s)=g_{2}(r)$ and therefore $r=s=0$.

In the calculations we shall frequently substitute arcs of curves by their chords. We will use the following two basic formulas for estimating the error.

$$
\lim _{h \rightarrow 0} \frac{\left|\frac{g_{i}(s+h)-g_{i}(s)}{\left|g_{i}(s+h)-g_{i}(s)\right|} \times \dot{g}_{i}(s)\right|}{h}=\frac{\kappa_{i}(s)}{2} \quad \text { and } \quad \lim _{h \rightarrow 0} \frac{\left|g_{i}(s+h)-g_{i}(s)\right|-h}{h^{2}}=\frac{\kappa_{i}(s)}{6} .
$$

We shall now estimate $s_{i+1}$ and $r_{i}$ in terms of $s_{i}$. First we take the triangles $g_{1}\left(s_{i}\right) g_{2}\left(r_{i}\right) X$ and $g_{1}\left(s_{i+1}\right) g_{2}\left(r_{i}\right) X$ the vertexes of which belong to the arcs we are interested in. The sine law combined with the above equations gives

$$
\frac{r_{i}}{s_{i}} \frac{\left(1+r_{i} O(1)\right)}{\left(1+s_{i} O(1)\right)}=\frac{\sin \omega_{i}+s_{i} O(1)}{\sin \left(\omega_{i}-\gamma\right)+s_{i} O(1)+r_{i} O(1)}
$$

and

$$
\frac{r_{i}}{s_{i+1}} \frac{\left(1+r_{i} O(1)\right)}{\left(1+s_{i+1} O(1)\right)}=\frac{\sin \left(\omega_{i+1}-\nu_{1}\left(s_{i+1}\right)\right)+s_{i+1} O(1)}{\sin \left(\omega_{i+1}-\nu_{1}\left(s_{i+1}\right)-\gamma\right)+s_{i+1} O(1)+r_{i} O(1)} .
$$

Geom. Dedicata, 59 (1996), 113-125.

(c) Á. Kurusa 
Because $r_{i}$ and $s_{i}$ approach the zero and $\left|\omega_{i}-\beta\right|<\delta_{0}$, these imply that

$$
\frac{s_{i+1}}{s_{i}}=\frac{\sin \left(\omega_{i+1}-\nu_{1}\left(s_{i+1}\right)-\gamma\right) \sin \omega_{i}}{\sin \left(\omega_{i+1}-\nu_{1}\left(s_{i+1}\right)\right) \sin \left(\omega_{i}-\gamma\right)}+s_{i} O(1)+s_{i+1} O(1)+r_{i} O(1)
$$

and

(6)

$\frac{r_{i+1}}{r_{i}}=\frac{\sin \left(\omega_{i+1}-\nu_{1}\left(s_{i+1}\right)-\gamma\right) \sin \omega_{i+1}}{\sin \left(\omega_{i+1}-\nu_{1}\left(s_{i+1}\right)\right) \sin \left(\omega_{i+1}-\gamma\right)}+s_{i} O(1)+s_{i+1} O(1)+r_{i} O(1)+r_{i+1} O(1)$

For $\delta_{0}$ and $\varepsilon_{1}$ chosen small enough, the convergence of $\omega_{i}$ follows from these equations in the following way.

Estimating $\omega_{i+1}$ by $\beta$ and $\nu_{1}\left(s_{i+1}\right)$ by $\beta-\alpha,(5)$ reads

$$
\left|\frac{s_{i+1}}{s_{i}}-\frac{\sin (\alpha-\gamma) \sin \beta}{\sin \alpha \sin (\beta-\gamma)}\right|<\delta_{0} O(1)+\varepsilon_{1} O(1)
$$

for $i$ big enough. By (1)

$$
\frac{\sin (\alpha-\gamma) \sin \beta}{\sin \alpha \sin (\beta-\gamma)}=1-\frac{\sin \gamma \sin \nu}{\sin \alpha \sin (\beta-\gamma)}
$$

where the right hand side is in $(0,1)$ clearly. Therefore $\lim \sup _{i \rightarrow \infty} \frac{s_{i+1}}{s_{i}}<1$ and in the same way $\lim \sup _{i \rightarrow \infty} \frac{r_{i+1}}{r_{i}}<1$. Then (2) implies that $\omega_{i}$ is Cauchy sequence and therefore convergent. From this the convergence of $r_{i} / s_{i}, r_{i} / s_{i+1}, s_{i+1} / s_{i}$ and $r_{i+1} / r_{i}$ follows via $(3),(4),(5)$ and $(6)$.

In conclusion we proved that the sequence of the straight lines $l_{2 i}$ tends to a straight line through $X$, hence the map $f$ is well defined (if $\delta_{0}$ and $\varepsilon_{1}$ is small enough).

By $\left(2^{\prime}\right)$ the angle of $f\left(l_{0}\right)$ to $t_{1}(0)$ is given by

$$
\bar{F}\left(\omega_{0}\right)=\omega_{0}+\sum_{i=0}^{\infty}\left(\nu_{1}\left(s_{i+1}\right)-\nu_{2}\left(r_{i}\right)\right)-\int_{s_{0}}^{0} \kappa_{1}(s) \mathrm{d} s
$$

for $\omega_{0} \in S\left(s_{0}\right)$. We are more interested in $\bar{F}\left(\omega_{0}\right)-\beta$, therefore we introduce $\xi_{i}=\omega_{i}-\beta_{1}\left(s_{i}\right)$ for $i=0,1,2, \ldots$ and set $F\left(\xi_{0}\right)=\lim _{i \rightarrow \infty} \xi_{i}=\bar{F}\left(\omega_{0}\right)-\beta$. We have

$$
F\left(\xi_{0}\right)=\xi_{0}+\sum_{i=0}^{\infty}\left(\nu_{1}\left(s_{i+1}\right)-\nu_{2}\left(r_{i}\right)\right)-\int_{s_{0}}^{0} \kappa_{1}(s) \mathrm{d} s+\beta_{1}\left(s_{0}\right)-\beta
$$

To conclude the proof we need to show that $F(\xi)=0$ implies $\xi=0$. This is the main point in the proof.

Geom. Dedicata, 59 (1996), 113-125.

(c) Á. Kurusa 
We shall trace the change of $\xi_{i}$ step-by-step. The equation

$$
\xi_{i+1}=\xi_{i}-\nu_{2}\left(r_{i}\right)+\nu_{1}\left(s_{i+1}\right)+\beta_{1}\left(s_{i}\right)-\beta_{1}\left(s_{i+1}\right)-\int_{s_{i}}^{s_{i+1}} \kappa_{1}(s) \mathrm{d} s
$$

comes from $\left(2^{\prime}\right)$. Let $g_{2}\left(\varrho_{i}\right)$ be the intersection of $g_{2}$ and $\mathcal{T}_{1}^{b}\left(s_{i}\right)$ and let $g_{1}\left(\sigma_{i+1}\right)$ be the intersection of $g_{1}$ and $\mathcal{T}_{2}^{a}\left(\varrho_{i}\right)$. Then we have

$$
\beta_{1}\left(s_{i}\right)=\beta_{1}\left(\sigma_{i+1}\right)-\nu_{1}\left(\sigma_{i+1}\right)+\nu_{2}\left(\varrho_{i}\right)-\int_{\sigma_{i+1}}^{s_{i}} \kappa_{1}(s) \mathrm{d} s .
$$

Combination of the two equations above results in

$$
\begin{aligned}
\xi_{i+1}=\xi_{i}+\left(\nu_{2}\left(\varrho_{i}\right)-\nu_{2}\left(r_{i}\right)\right) & +\left(\nu_{1}\left(s_{i+1}\right)-\nu_{1}\left(\sigma_{i+1}\right)\right)+ \\
& +\left(\beta_{1}\left(\sigma_{i+1}\right)-\beta_{1}\left(s_{i+1}\right)\right)-\int_{\sigma_{i+1}}^{s_{i+1}} \kappa_{1}(s) \mathrm{d} s .
\end{aligned}
$$

We estimate the terms on the right hand side one-by-one in terms of $\xi_{i}$.

Let $x_{i}=\left|g_{1}\left(s_{i}\right) g_{2}\left(r_{i}\right)\right|$ and $\gamma_{i}=g_{1}\left(s_{i}\right) X g_{2}\left(r_{i}\right) L$. Using the sine law for $\frac{\varrho_{i}-r_{i}}{x_{i}}$ and $\frac{r_{i}}{x_{i}}$ in the triangles $g_{1}\left(s_{i}\right) g_{2}\left(r_{i}\right) g_{2}\left(\varrho_{i}\right)$ and $g_{1}\left(s_{i}\right) g_{2}\left(r_{i}\right) X$, respectively, (approximating the arcs with their respective chords) and then dividing the results we obtain that

$$
\frac{\varrho_{i}-r_{i}}{r_{i}} \frac{1+\left(\varrho_{i}-r_{i}\right) O(1)}{1+r_{i} O(1)}=\frac{\sin \xi_{i} \sin \gamma_{i}}{\sin \beta_{2}\left(\varrho_{i}\right)\left(\sin \omega_{i}+s_{i} O(1)\right)} .
$$

Since $\gamma_{i} \rightarrow \gamma, \omega_{i} \rightarrow \bar{F}\left(\omega_{0}\right)$ and $\beta_{2}\left(\varrho_{i}\right) \rightarrow \beta-\gamma,(10)$ can be written as

$$
\varrho_{i}=r_{i}+\xi_{i} r_{i} K_{i}
$$

where $\lim _{i \rightarrow \infty} K_{i}=K_{\infty}$ is finite. In the second order Taylor expansion of $\nu_{2}$ this gives

$$
\nu_{2}\left(\varrho_{i}\right)=\nu_{2}\left(r_{i}\right)+\dot{\nu}_{2}\left(r_{i}\right) \xi_{i} r_{i} K_{i}+\xi_{i}^{2} r_{i}^{2} O(1)
$$

For the other terms on the right hand side of $(9)$, we need to estimate $\frac{\sigma_{i+1}-s_{i+1}}{s_{i+1}}$. Let $y_{i}=\left|g_{2}\left(r_{i}\right) g_{1}\left(s_{i+1}\right)\right|$ and $\bar{\gamma}_{i}=g_{1}\left(s_{i+1}\right) X g_{2}\left(r_{i}\right) \angle$. Let $I_{i}=$ $l_{2 i+1} \cap \tau_{2}^{a}\left(\varrho_{i}\right)$ and $z_{i}=\left|I_{i} g_{1}\left(s_{i+1}\right)\right|$. The sine law for the triangle $g_{1}\left(s_{i+1}\right) g_{2}\left(r_{i}\right) X$ gives

$$
\frac{y_{i}}{s_{i+1}\left(1+s_{i+1} O(1)\right)}=\frac{\sin \bar{\gamma}_{i}}{\sin \left(\omega_{i+1}-\nu_{i}\left(s_{i+1}\right)-\bar{\gamma}_{i}\right)+s_{i+1} O(1)}
$$

Geom. Dedicata, 59 (1996), 113-125.

(c) Á. Kurusa 
Again the sine law, but in the triangles $g_{1}\left(\sigma_{i+1}\right) g_{1}\left(s_{i+1}\right) I_{i}$ and $g_{2}\left(\varrho_{i}\right) g_{2}\left(r_{i}\right) I_{i}$ leads to

$\left(13^{\prime \prime}\right) \quad \frac{\left(\sigma_{i+1}-s_{i+1}\right)\left(1+\left(\sigma_{i+1}-s_{i+1}\right) O(1)\right)}{z_{i}}=\frac{\sin \left(\xi_{i}+\nu_{2}\left(r_{i}\right)-\nu_{2}\left(\varrho_{i}\right)\right)}{\sin \alpha_{1}\left(\sigma_{i+1}\right)+\left(\sigma_{i+1}-s_{i+1}\right) O(1)}$

and

$$
\frac{\left(\varrho_{i}-r_{i}\right)\left(1+\left(\varrho_{i}-r_{i}\right) O(1)\right)}{z_{i}+y_{i}}=\frac{\sin \left(\xi_{i}+\nu_{2}\left(r_{i}\right)-\nu_{2}\left(\varrho_{i}\right)\right)}{\sin \alpha_{2}\left(\varrho_{i}\right)+\left(\varrho_{i}-r_{i}\right) O(1)}
$$

where $\xi_{i}+\nu_{2}\left(r_{i}\right)-\nu_{2}\left(\varrho_{i}\right)$ is the angle between $l_{2 i+1}$ and $\tau_{2}^{a}\left(\varrho_{i}\right)$ at $I_{i}$. Eliminating $z_{i}$ and $y_{i}$ from $\left(13^{\prime \prime \prime}\right)$ with $\left(13^{\prime \prime}\right)$ and $\left(13^{\prime}\right)$ gives us that

$$
\begin{aligned}
& \frac{\left(\sigma_{i+1}-s_{i+1}\right)}{s_{i+1}}\left(\sin \alpha_{1}\left(\sigma_{i+1}\right)+\left(\sigma_{i+1}-s_{i+1}\right) O(1)\right) \\
& =\frac{\left(\varrho_{i}-r_{i}\right)}{r_{i}} \frac{r_{i}}{s_{i+1}}\left(\sin \alpha_{2}\left(\varrho_{i}\right)+\left(\varrho_{i}-r_{i}\right) O(1)\right)- \\
& \quad-\xi_{i} \frac{\sin \left(\xi_{i}+\nu_{2}\left(r_{i}\right)-\nu_{2}\left(\varrho_{i}\right)\right)}{\xi_{i}} \frac{\sin \bar{\gamma}_{i}}{\sin \left(\omega_{i+1}-\nu_{i}\left(s_{i+1}\right)-\bar{\gamma}_{i}\right)+s_{i+1} O(1)}
\end{aligned}
$$

The terms $\left(\varrho_{i}-r_{i}\right) / r_{i}$ and $r_{i} / s_{i+1}$ are convergent by (11) and (4). We also know

$$
\frac{\sin \left(\xi_{i}-\nu_{2}\left(r_{i}\right)+\nu_{2}\left(\varrho_{i}\right)\right)}{\xi_{i}} \longrightarrow \begin{cases}\frac{\sin F\left(\xi_{0}\right)}{F\left(\xi_{0}\right)} & \text { if } F\left(\xi_{0}\right) \neq 0 \\ 1 & \text { if } F\left(\xi_{0}\right)=0\end{cases}
$$

from equation (12). Therefore (13) can be written as

$$
\sigma_{i+1}=s_{i+1}+\xi_{i} s_{i+1} L_{i}
$$

where $\lim _{i \rightarrow \infty} L_{i}=L_{\infty}$ is finite. As to the terms in (9) this implies the Taylor expansions of second order

$$
\begin{aligned}
\nu_{1}\left(\sigma_{i+1}\right) & =\nu_{1}\left(s_{i+1}\right)+\dot{\nu}_{1}\left(s_{i+1}\right) \xi_{i} s_{i+1} L_{i}+\xi_{i}^{2} s_{i+1}^{2} O(1), \\
\beta_{1}\left(\sigma_{i+1}\right) & =\beta_{1}\left(s_{i+1}\right)+\dot{\beta}_{1}\left(s_{i+1}\right) \xi_{i} s_{i+1} L_{i}+\xi_{i}^{2} s_{i+1}^{2} O(1), \\
\int_{s_{i+1}}^{\sigma_{i+1}} \kappa_{1}(s) \mathrm{d} s & =\kappa_{1}\left(s_{i+1}\right) \xi_{i} s_{i+1} L_{i}+\xi_{i}^{2} s_{i+1}^{2} O(1) .
\end{aligned}
$$

Using these and (12) in (9), and ordering the result according to the power of $\xi_{i}$, we obtain

$$
\xi_{i+1}=\xi_{i}\left(1+s_{i+1} N_{i+1}+\xi_{i} s_{i+1}^{2} O(1)\right)
$$

Geom. Dedicata, 59 (1996), 113-125.

(c) Á. Kurusa 
where

$$
N_{i+1}=\dot{\nu}_{2}\left(r_{i}\right) \frac{r_{i}}{s_{i+1}} K_{i}-\dot{\nu}_{1}\left(s_{i+1}\right) L_{i}+\dot{\beta}_{1}\left(s_{i+1}\right) L_{i}+\kappa_{1}\left(s_{i+1}\right) L_{i}
$$

and hence $\lim _{i \rightarrow \infty} N_{i}=N_{\infty}$ is finite. Let $h_{i+1}=s_{i+1} N_{i+1}+\xi_{i} s_{i+1}^{2} O(1)$. Then from (19) we have

$$
F\left(\xi_{0}\right)=\xi_{0} \prod_{i=1}^{\infty}\left(1+h_{i}\right)
$$

We know that the infinite product exists, and we have to prove it is not zero.

Assume $F\left(\xi_{0}\right)=0$. Then the limits $K_{\infty}$ and $L_{\infty}$ becomes easy to determine: From (10) we obtain $K_{\infty}$, and then (13) gives $L_{\infty}$ using $K_{\infty}$, (4) and (14). The results are

$$
K_{\infty}=\frac{\sin \gamma}{\sin \beta \sin (\beta-\gamma)} \quad \text { and } \quad L_{\infty}=\frac{\sin \gamma}{\sin \alpha \sin (\alpha-\gamma)}-\frac{\sin \gamma}{\sin \beta \sin (\beta-\gamma)}
$$

Substituting these, (4), the first part of the lemma's formula and the second part of $(*)$ into $(20)$ one easily computes

$$
N_{\infty}=\lim _{i \rightarrow \infty} N_{i+1}=\frac{(a \sin \alpha-b \sin \beta) \sin \gamma}{a b \sin \beta \sin (\alpha-\gamma)}
$$

By our initial condition this is not zero, hence

$$
\lim _{i \rightarrow \infty} \frac{h_{i+1}}{h_{i}}=\lim _{i \rightarrow \infty} \frac{s_{i+1} N_{i+1}+\xi_{i} s_{i+1}^{2} O(1)}{s_{i} N_{i}+\xi_{i-1} s_{i}^{2} O(1)}=\lim _{i \rightarrow \infty} \frac{s_{i+1}}{s_{i}}<1 .
$$

This implies $\prod_{i=1}^{\infty}\left(1+h_{i}\right) \neq 0$ and therefore $F(\xi)=0$ implies $\xi=0$.

Thus we have proved that the only solution of the equation $F(\xi)=0$ is $\xi=0$. Since $F$ is constructed from the known visual angle functions, $F$ is known. Therefore we can determine the only straight line $l$ in $S\left(s_{0}\right)$, that is $\mathcal{T}_{1}^{b}\left(s_{0}\right)$ of course, for which $f(l)$ is the tangent $\mathcal{T}_{1}^{b}(0)$. This completes the proof.

Note that in proving $F(\xi) / \xi \neq 0$ we heavily used the existence of $F\left(\xi_{0}\right)=$ $\lim _{i \rightarrow \infty} \xi_{i}$, hence the fore-part of the proof can not be omitted, although it is implied by the latter part.

Now we are ready to prove our main result.

Geom. Dedicata, 59 (1996), 113-125.

(c) Á. Kurusa 
Proof of Theorem 1. Suppose that $\mathcal{F}_{1} \not \equiv \mathcal{F}_{2}$. Since their visual angle functions are equal, neither of them can contain the other one, hence they have a common tangent, say $\mathcal{T}_{0}$.

Our first step is to prove that there are two common tangents of $\mathcal{F}_{1}$ and $\mathcal{F}_{2}$ intersecting each other in a point of $\partial \mathcal{C}_{1} \cap \partial \mathcal{C}_{2}$. We separate two cases.

Case I. If $\mathcal{T}_{0}$ goes through a point $X$ of $\partial \mathcal{C}_{1} \cap \partial \mathcal{C}_{2}$, then we have an other common tangent through $X$, because $\mathcal{F}_{1}$ and $\mathcal{F}_{2}$ subtend equal angles at $X$.

Case II. If $\mathcal{T}_{0}$ does not go through a point of $\partial \mathcal{C}_{1} \cap \partial \mathcal{C}_{2}$ then it intersects a component of $\operatorname{Int}\left(\mathcal{C}_{1} \triangle \mathcal{C}_{2}\right)$. This component is bounded by two arcs, one of $\mathcal{C}_{1}$ and the other of $\mathcal{C}_{2}$. Let us denote the two intersections of these two arcs by $X$ and $Y$. We show a sequence of common tangents of $\mathcal{F}_{1}$ and $\mathcal{F}_{2}$ so that its limits will be straight lines through $X$ and $Y$. Since the set of the common tangents is topologically closed, this implies the existence of common tangents through $X$ and $Y$.

Let us suppose that the intersections $\mathcal{T}_{0} \cap \mathcal{F}_{1}$ and $\mathcal{T}_{0} \cap \partial \mathcal{C}_{2}$ are separated by $\mathcal{T}_{0} \cap \partial \mathcal{C}_{1}$. Let $X_{i}=\mathcal{T}_{2 i} \cap \partial \mathcal{C}_{1}$ and $Y_{i}=\mathcal{T}_{2 i} \cap \partial \mathcal{C}_{2}$. There must be an other common tangent $\mathcal{T}_{2 i+1}$ of $\mathcal{F}_{1}$ and $\mathcal{F}_{2}$ through $Y_{i}$. Define $X_{i+1}=\mathcal{T}_{2 i+1} \cap \partial \mathcal{C}_{1}$. Then again a new common tangent through $X_{i+1}$ exists and we call it $\mathcal{T}_{2 i+2}$. Let $Y_{i+1}=\mathcal{T}_{2 i+2} \cap \partial \mathcal{C}_{2}$. Suppose that $X_{i+1}$ separates $X_{i}$ and $X$ on the $\operatorname{arc} X Y \subset \partial \mathcal{C}_{1}$. Then also $Y_{i+1}$ separates $Y_{i}$ and $X$ on $X Y \subset \partial \mathcal{C}_{2}$. The previous theorem says $X_{i} \rightarrow X, Y_{i} \rightarrow X$ and $\mathcal{T}_{2 i}$ resp. $\mathcal{T}_{2 i+1}$ converge to two common tangents through $X$.

Summing up, we have a point $X \in \partial \mathcal{C}_{1} \cap \partial \mathcal{C}_{2}$ with two common tangents of $\mathcal{F}_{1}$ and $\mathcal{F}_{2}$. Our proposition now implies that $\mathcal{F}_{1}$ and $\mathcal{F}_{2}$ have an open set of common tangents through any point of $\partial \mathcal{C}_{1}$ and $\partial \mathcal{C}_{2}$ sufficiently close to $X$. We call this neighborhood of $X$ the good neighborhood.

To conclude the proof we show that the set of the common tangents is open (the topology is simply given by the topology of the normal vectors in the circle). Since it is at once closed, this proves the theorem.

There exist two cases again. Let $\mathcal{T}_{0}$ be a common tangent. If it goes through a point of $\partial \mathcal{C}_{1} \cap \partial \mathcal{C}_{2}$ we have the good neighborhood of $\mathcal{T}_{0} \cap \partial \mathcal{C}_{1} \cap \partial \mathcal{C}_{2}$.

In the second case we have to "move" the good neighborhood of $X$ to $X_{0}=$ $\mathcal{T}_{0} \cap \partial \mathcal{C}_{1}$. Create the sequence $X_{i}$ as above. This converges to $X$, therefore there is an $X_{k}$ in the good neighborhood of $X$. Then $\mathcal{T}_{2 k}$ has a neighborhood of common tangents. Obviously, if $\mathcal{T}_{j}$ has a neighborhood of common tangents, then also $\mathcal{T}_{j-1}$ has such neighborhood, hence the theorem is proved.

Note that $\mathcal{C}_{1}$ and $\mathcal{C}_{2}$ are not necessarily compact in the theorem. For example, the statement is valid for two non-parallel half planes as well! Also note, that the proof does not work without the $\gamma \neq 0$ condition.

Geom. Dedicata, 59 (1996), 113-125.

(c) Á. Kurusa 


\section{Some generalizations}

The most natural generalization is to consider the problem in higher dimensions, but we have more ways to define 'visual angle'. In two dimension the shape of the visual angle and its measure cover the same information, but in the higher dimensions this holds no longer. Moreover, in higher dimensions one can choose further possibilities using different dimensions for the picture.

Let $n$ be the dimension and $1<k \leq n$. Let $\mathcal{D}^{n, k}=\left\{(P, K) \in \mathbb{R}^{n} \times G^{n, k}: P \in\right.$ $K\}$, where $G^{n, k}$ is the Grassman manifold of the $k$-dimensional hyperplanes. We call the set $V_{\mathcal{F}}(P) \subseteq S^{k-1}$ the visual shape of a convex body $\mathcal{F}$ at $(P, K) \in \mathcal{D}^{n, k}$ if there is an isometric map $m$ of $S^{k-1}$ into $K$ so that the center of $m\left(S^{k-1}\right)$ is $P$ and for any $\omega \in S^{k-1}$ the straight line $\{P+\lambda \omega: \lambda \in \mathbb{R}\}$ intersect $\mathcal{F}$ if and only if $\omega \in V_{\mathcal{F}}(P)$. The surface measure of $V_{\mathcal{F}}(P)$ is called the visual angle of $\mathcal{F}$ at $(P, K) \in \mathcal{D}^{n, k}$.

Theorem 2. Let $n>2$ and $1<k<n$. Let $\mathcal{C}$ and $\mathcal{F}$ be convex bodies, $\mathcal{F} \subset \operatorname{Int} \mathcal{C}$. Then $\mathcal{F}$ can be reconstructed by its $(k-1)$-dimensional visual angles at $(P, K)$, where $P \in \partial \mathcal{C}$.

Proof. Take a point $P$ of $\partial \mathcal{C}$. Let $\mathcal{F}_{P}$ be the intersection of $\mathrm{S}_{P}^{n-1}$, the unit sphere centered at $P$, with $\cup_{X \in \mathcal{F}} \overline{X P}$, where $\overline{X P}$ is the straight line through $X$ and $P$. Since $\mathcal{F}$ is compact and convex, $\mathcal{F}_{P}$ is contained in a half sphere of $\mathrm{S}^{n-1}$. Then a $(k-1)$-dimensional visual angle at $(P, K)$ is the integral of the indicator function $\chi$ of $\mathcal{F}_{P}$ over the $(k-1)$-dimensional great sphere $K \cap \mathrm{S}^{n-1}$. Therefore, the visual angle function at the point $P$ as it depends on $K$ is just the $(k-1)$-dimensional Radon transform of $\chi$. It is very well known [11] that this transform is invertible. The inversion of the Radon transform therefore gives back the $(n-1)$-dimensional visual shape of $\mathcal{F}$ together with its position at every points of $\partial \mathcal{C}$. Obviously these determine $\mathcal{F}$ that was to be proved.

This surprisingly easy theorem leaves unanswered the uniqueness problem only for the $(n-1)$-dimensional visual shape and of the $(n-1)$-dimensional visual angle (if affirmative, this would imply the previous one). T. Ódor could generalize Nietsche's result for higher dimension and it turned out, that for $n \geq 3$ one sphere is enough to distinguish any concentric sphere from the other convex bodies [9].

Another interesting generalization in the plane comes from a connection with the problem G.8 of [1]. Let $n(\omega, X)$ be the number of the intersections of the curve $c$ with the straight line $l(\omega, X)$ going through $X$ in direction $\omega \in \mathrm{S}^{1}$. The question

Geom. Dedicata, 59 (1996), 113-125.

(c) Á. Kurusa 
is if the function $n$ determines the curve $c$. Horwitz proved in [5], that a $\mathrm{C}^{2}$ curve is determined by the function $n$. (Note that $n(\omega, X)=n(\omega, X+t \omega)=n(-\omega, X)$ for any $t \in \mathbb{R}$.)

Let the $\mathrm{C}^{2}$ curve $c$ be the boundary of a convex body $\mathcal{C}$. Then the function $n$ determines the visual angle $\nu$ of $\mathcal{C}$ at any point $X$ by

$$
\nu(X)=\frac{1}{4} \int_{\mathrm{S}^{1}} n(\omega, X) \mathrm{d} \omega .
$$

Therefore our result strengthens Horwitz's result for convex curves. On the other hand, (22) offers a very natural way to extend the definition of the visual angle for arbitrary curves.

Admitting (22) as the definition of the visual angle for general curves we may ask for the uniqueness again. Horwitz's and our results indicate that the distinction between any two curves by their visual angles (22) will need a two dimensional set of points. We formulate the following

Conjecture. Let $\mathcal{C}_{1}$ and $\mathcal{C}_{2}$ be compact domains so that $\mathcal{C}_{1} \subset \operatorname{Int} \mathcal{C}_{2} . f_{1}$ and $f_{2}$ are $\mathrm{C}^{2}$ curves of finite length and $f_{1} \cup f_{2} \subset \operatorname{Int} \mathcal{C}_{1}$. If the visual angles $\nu_{1}$ and $\nu_{2}$ of $f_{1}$ and $f_{2}$ are equal at each point of $\operatorname{Int} \mathcal{C}_{2} \backslash \mathcal{C}_{1}$, then $f_{1}$ and $f_{2}$ coincide.

\section{References}

[1] H. T. Craft, K. J. Falconer and R. K. Guy, Unsolved problems in geometry, Springer-Verlag, New York, 1991.

[2] K. J. Falconer, X-ray problems for point sources, Proc. London Math. Soc., 46 (1983), 241-262.

[3] R. J. Gardner and P. McMullen, On Hammer's X-ray problem, J. London Math. Soc., 21 (1980), 171-175.

[4] J. W. Green, Sets subtending a constant angle on a circle, Duke Math. J., 17 (1950), 263-267.

[5] A. Honwitz, Reconstructing a function from its tangent lines, Amer. Math. Monthly, 96 (1989), 807-813.

[6] J. Kincses and Á. Kurusa, Can you recognize the shape of a figure from its shadows?, Beiträge zur Alg. und Geom., 36 (1995), 25-34.

[7] Á. Kurusa, The shadow picture problem for nonintersecting curves, Geom. Dedicata, 59 (1996), 113-125.

[8] J. C. C. Nietsche, Isoptic characterization of a circle, (Proof of a conjecture of M.S. Klamkin), Amer. Math. Monthly, 97 (1990), 45-47.

[9] T. Ódor, Oral discussion.

[10] C. A. Rogers, An equichordal problem, Geom. Dedicata, 10 (1981), 73-78.

Geom. Dedicata, 59 (1996), 113-125.

(c) Á. Kurusa 
[11] R. Schneider, Functions on a sphere with vanishing integrals over certain subspheres, J. of Math. Anal. and Appl., 26 (1969), 381-384.

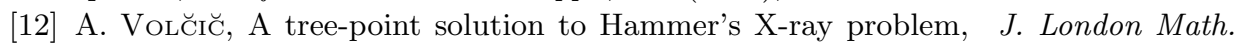
Soc., 34 (1986), 349-359.

Á. Kurusa, Bolyai Institute, Aradi vértanúk tere 1., H-6720 Szeged, Hungary; e-mail: kurusa@math.u-szeged.hu 